\title{
A SOLUTION OF THE HEAT TRANSFER EQUATION FOR LAMINAR FLOW BETWEEN PARALLEL PLATES*
}

BT

\author{
S. C. R. DENNIS AND G. POOTS
}

The Queen's University of Belfast

Introduction. Consider a viscous incompressible fluid flowing from left to right between infinite parallel boundary walls. The walls have a temperature $\theta_{0}$ to the left of the origin and zero to the right of it. If the heat transmissivity of the walls is taken to be negligible compared with that of the fluid and heat dissipation within the fluid is neglected, then for Poiseuille flow the dimensionless reduced temperature $\vartheta\left(=\theta / \theta_{0}\right)$ satisfies the equation

$$
\kappa\left(\frac{\partial^{2} \vartheta}{\partial \xi^{2}}+\frac{\partial^{2} \vartheta}{\partial \eta^{2}}\right)=u \frac{\partial \vartheta}{\partial \xi}
$$

with

$$
u(\eta)=\frac{3}{2} u_{0}\left(1-\frac{\eta^{2}}{D^{2}}\right),
$$

where $\kappa$ is the thermometric conductivity, $u$ the longitudinal velocity component, $u_{0}$ the mean velocity and $2 D$ the distance between the plates. If we introduce the dimensionless variables $x$ and $y$ defined by $\xi=\frac{3}{2} \mathrm{P} e D x, \eta=D(2 y / \pi-1)$ then with the substitution (2), Eq. (1) becomes

$$
\alpha^{2} \cdot \frac{\partial^{2} \vartheta}{\partial x^{2}}+\frac{\partial^{2} \vartheta}{\partial y^{2}}=\frac{16}{\pi^{3}}\left(y-\frac{y^{2}}{\pi}\right) \frac{\partial \vartheta}{\partial x}, \quad \alpha=\frac{4}{3 \pi \mathrm{Pe}}
$$

and if the fluid to the left of the origin is assumed to have the same temperature as the plates, the boundary conditions are

$$
\begin{aligned}
& \vartheta=0 \text { for } x>0, \quad y=0 \text { or } \pi, \\
& \vartheta=1 \text { for } x=0 \text { and } \vartheta \rightarrow 0 \text { as } x \rightarrow \infty, \text { for } 0<y<\pi .
\end{aligned}
$$

The parameter Pé is the Péclet number and may be quite large, even for moderately small Reynolds numbers, so that for most practical purposes the first term in (3a) may be omitted. In fact in any case in which this term is not omitted, it is probably not justifiable to neglect the heat dissipation term in formulating (1). This term involves no significant change in the present mode of solution, however, and will not be considered further. It should also be noted that, with the above simplification in (3a), and taking $x$ as a time coordinate, this equation assumes a particular form of the heat conduction equation for one-dimensional heat flow in a medium whose thermal properties vary with position (but not with time or temperature).

This paper is not concerned with thermal properties, but with a mathematical method of solution of these problems. The solution of (3a), subject to the conditions (3b), has been obtained when $\alpha$ is small by Prins, Mulder and Schenk [1], who solved using power

*Received June 21, 1955. 
series the corresponding Sturm-Liouville equation obtained on separation of variables. The method appears to be laborious, however, and our object is to indicate a new approach in which formal Fourier analysis, of the type developed by Carslaw and Jaeger [2], may be extended to problems of this kind. A detailed solution is given in the case of (3a) and the method is then extended to the general case in which $u(\eta)$ may be any function satisfying Dirichlet's conditions. It is capable of extension to three-dimensional problems, and, in particular, to the problem of forced heat convection through a duct with rectangular cross-section.

Solution of the heat transfer equation by Fourier analysis. From consideration of the boundary conditions ( $3 \mathrm{~b})$ the local temperature distribution is symmetrical about $y=\pi / 2$ and is zero at $y=0$ or $\pi$ for $x>0$, so that we may expand $\vartheta(x, y)$ as the Fourier series,

$$
\vartheta(x, y)=\frac{2}{\pi} \sum_{n=1}^{\infty} v_{n}(x) \sin n y \quad(n=1,3,5, \cdots) .
$$

Then in the usual manner, multiplying each side of (3a) by $\sin n y$ and integrating with respect to $y$ from $y=0$ to $y=\pi$ we obtain

$$
\alpha^{2} \frac{d^{2} v_{n}}{d x^{2}}-n^{2} v_{n}=\frac{16}{\pi^{3}} \int_{0}^{\pi}\left(y-\frac{y^{2}}{\pi}\right) \frac{\partial \vartheta}{\partial x} \sin n y d y
$$

with

$$
v_{n}(0)=\frac{2}{n}, \quad v_{n}(\infty)=0 .
$$

Putting

$$
y-\frac{y^{2}}{\pi}=\frac{1}{2} b_{0}+\sum_{q=2}^{\infty} b_{q} \cos q y \quad(q=2,4,6, \cdots),
$$

where

$$
b_{0}=\frac{\pi}{3}, \quad b_{a}=-\frac{4}{\pi q^{2}},
$$

then we find

$\int_{0}^{\pi}\left(y-\frac{y^{2}}{\pi}\right) \frac{\partial \vartheta}{\partial x} \sin n y d y=\left(\frac{\pi}{6}+\frac{1}{2 \pi n^{2}}\right) \frac{d v_{n}}{d x}-\frac{8 n}{\pi} \sum_{p=1}^{\infty} \frac{p}{\left(n^{2}-p^{2}\right)^{2}} \frac{d v_{p}}{d x} \quad(n \neq p)$,

where the summation extends only to odd values of $p$.

Finally we arrive at the infinite set of simultaneous constant coefficient equations in $v_{n}(x)$ given by

$$
\alpha^{2} \frac{d^{2} v_{n}}{d x^{2}}-\left(\frac{8}{3 \pi^{2}}+\frac{8}{\pi^{4} n^{2}}\right) \frac{d v_{n}}{d x}+\frac{128 n}{\pi^{4}} \sum_{p=1}^{\infty} \frac{p}{\left(n^{2}-p^{2}\right)^{2}} \frac{d v_{p}}{d x}(n \neq p)-n^{2} V_{n}=0
$$

and their solution is required subject to the conditions (5a). This system will be satisfied by

if

$$
v_{n}(x)=A_{n} \exp (\lambda x)
$$

$$
f(\lambda, n) A_{n}+\frac{128 \lambda n}{\pi^{4}} \sum_{p=1}^{\infty} \frac{p A_{p}}{\left(n^{2}-p^{2}\right)^{2}}(n \neq p)=0,
$$


where

$$
f(\lambda, n) \equiv \alpha^{2} \lambda^{2}-\left(\frac{8}{3 \pi^{2}}+\frac{8}{\pi^{4} n^{2}}\right) \lambda-n^{2}
$$

and $\lambda$ is obtained from the condition for non-vanishing $A_{n}$. This condition may be written compactly as

$$
\Delta(\lambda) \equiv\left|a_{i j}(\lambda)\right|=0 \quad(i, j=1,3,5, \cdots),
$$

where

$$
a_{i j}=a_{i i}=\frac{128 \lambda}{\pi^{4}} \frac{i j}{\left(i^{2}-j^{2}\right)^{2}}(i \neq j), \quad a_{i i}=f(\lambda, i) .
$$

The diagonal elements of (11) are very much larger, by virtue of the term $i^{2}$, than the non-diagonal elements [i.e. $a_{i i}(\lambda) \gg a_{i j}(\lambda)$ ] thus as a first approximation to the solution of (11) we assume that

$$
\left|a_{i j}(\lambda)\right| \bumpeq \prod_{m=1}^{\infty} a_{m m}(\lambda)
$$

giving approximately the $m$ th $\operatorname{root} \lambda_{m}$ to be the solution of $f\left(\lambda_{m}, m\right)=0$. This equation has two real roots of opposite sign but only the negative one is acceptable on account of (5a). Corresponding to each $\lambda_{m}$ for $m=1,3,5 \cdots$ there is an associated set of $A_{n}^{(m)}$ for $n=1,3,5 \cdots$ and using the above approximation as an initial estimate of $\lambda_{m}$ a rapid process of successive approximation may be set up for calculating the correct $\lambda_{m}$ and associated $A_{n}^{(m)}$ as follows. The $m$ th equation of (9) is used to determine $\lambda_{m}$. If we put $A_{m}$ arbitrarily equal to unity in (9) we obtain for $\lambda_{m}$ the equation

$$
f\left(\lambda_{m}, m\right)+\frac{128 m}{\pi^{4}} \lambda_{m} \sum_{p=1}^{\infty} \frac{p}{\left(m^{2}-p^{2}\right)^{2}} A_{p}^{(m)}(m \neq p)=0 .
$$

The remainder of (9), excluding $n=m$, may be written

$$
f\left(\lambda_{m}, n\right) A_{n}^{(m)}+\frac{128}{\pi^{4}} \lambda_{m} \sum_{p=1}^{\infty} \frac{p}{\left(n^{2}-p^{2}\right)^{2}} A_{p}^{(m)}(n \neq p)=-\frac{128}{\pi^{4}} \lambda_{m} \frac{m n}{\left(m^{2}-n^{2}\right)^{2}}
$$

and once $\lambda_{m}$ is given these may be used for determining the associated $A_{n}^{(m)}$. The process is now as follows. Taking the initial estimate of $\lambda_{m}$ as the negative root of $f\left(\lambda_{m}, m\right)=0$ we substitute this in (14). These equations are well conditioned because of the leading diagonal elements and very accurate estimates of the $A_{n}^{(m)}$ may be obtained by neglecting the terms under the summation sign giving

$$
A_{n}^{(m)} \bumpeq-\frac{128}{\pi^{4}} \lambda_{m} \frac{m n}{\left(n^{2}-n^{2}\right)^{2}} / f\left(\lambda_{m}, n\right) .
$$

From the estimates (15) the summations in (14) are computed and in this way we arrive at the $A_{n}^{(m)}$ corresponding to the assumed $\lambda_{m}$. We now return to (13) and re-calculate $\lambda_{m}$ and the process is repeated until sufficient accuracy is attained. In the numerical

*In order to attach any meaning to (11) it must first be shown that the infinite determinant $\Delta(\lambda)$ can be made convergent. Absolute convergence is readily achieved in this case by dividing each row by its corresponding leading diagonal element, provided that $\lambda$ is not such as to make any of these elements zero (see for example, Whittaker and Watson [3]). 
examples considered it was found that if we aim at five decimal accuracy two complete iterations were sufficient. For example, in the case of large Pé, when the first term of (3a) may be neglected, the initial estimate of $\lambda_{3}=-32.2217$. Using this value in (15) a set of $A_{n}^{(3)}$ was obtained which were then improved using (14). The second estimate of $\lambda_{3}=-32.1478$ and on using (14) again only minor corrections occurred. The final accepted value of $\lambda_{3}$ was found to be $\lambda_{3}=-32.1472$.

It remains now to calculate the constants of integration as these have so far been chosen arbitrarily by setting $A_{m}^{(m)}=1$. Thus, if we write

where

$$
\vartheta(x, y)=\frac{2}{\pi} \sum_{m=1}^{\infty} a_{m} \exp \left(\lambda_{m} x\right) X_{m}(y)
$$

$$
X_{m}(y)=\sum_{p=1}^{\infty} A_{p}^{(m)} \sin p y,
$$

then using the boundary condition $(3 \mathrm{~b})$ for $v_{n}(0)$ we have

$$
\sum_{m=1}^{\infty} A_{n}^{(m)} Q_{m}=\frac{2}{n} \quad(n=1,2,5, \cdots)
$$

so that (17) provide an infinite set of simultaneous equations for the calculation of the $a_{m}$. For computational purposes however, these are unsatisfactory because of the slow convergence of the right hand side and it is better to proceed as follows. We make use of the variational principle that the quantities $a_{m}$ must be so chosen that, when $x=0$, the integral

$$
J\left(a_{1}, a_{3}, \cdots\right)=\int_{0}^{\pi}\left(\sum_{n=1}^{\infty} a_{n} X_{n}-\Pi / 2\right)^{2} d y
$$

shall be a minimum. Thus for $m=1,3,5, \cdots$ we have

$$
\frac{\partial J}{\partial Q_{m}}=2 \int_{0}^{\pi}\left(\sum_{n=1}^{\infty} a_{n} X_{n}-\Pi / 2\right) X_{m} d y=0
$$

and on simplification of this integral we obtain the alternative set of simultaneous equations for the $Q_{m}$ given by

$$
2 \sum_{p=1}^{\infty} \frac{1}{p} A_{p}^{(n)}=\sum_{m=1}^{\infty} \alpha_{m}\left(\sum_{p=1}^{\infty} A_{p}^{(m)} A_{p}^{(n)}\right) \quad(n=1,3,5, \cdots) .
$$

Although (18) are more complicated, the derivation of the $a_{m}$ from them is in fact more accurate. In practice to quote the local temperature distribution in the regions of interest to within $0.1 \%$ it is necessary to obtain only the first four or five $\lambda_{m}$ since these occur in $\vartheta(x, y)$ as $\exp \left(\lambda_{m} x\right)$. In the special case of the foregoing for which the term $\alpha^{2} \partial^{2} \vartheta / \partial x^{2}$ may be neglected in (3a), explicit formulae may be derived for the $a_{m}$ on account of the orthogonality of the functions $X_{m}(y)$. Thus

$$
\int_{0}^{\pi}\left(y-\frac{y^{2}}{\pi}\right) X_{m}(y) X_{n}(y)=0 \text { for } m \neq n,
$$

so that

$$
\mathrm{Q}_{m}=\frac{\pi}{2} \int_{0}^{\pi}\left(y-\frac{y^{2}}{\pi}\right) X_{m}(y) d y / \int_{0}^{\pi}\left(y-\frac{y^{2}}{\pi}\right) X_{m}^{2}(y) d y
$$


and putting

$$
X_{m}(y)=\sum_{p=1}^{\infty} A_{p}^{(m)} \sin p y
$$

we obtain

$$
Q_{m}=\sum_{p=1}^{\infty} \frac{A_{p}^{(m)}}{p^{3}} /\left\{\frac{\pi^{2}}{24} \sum_{p=1}^{\infty}\left[A_{p}^{(m)}\right]^{2}-\frac{1}{2} \sum_{n=2}^{\infty} \frac{I_{n}^{(m)}}{n^{2}}\right\}
$$

where

$$
I_{n}^{(m)}=\sum_{p=1}^{\infty} \sum_{a=1}^{\infty} A_{p}^{(m)} A_{a}^{(m)}(n=|p-q|)-\sum_{p=1}^{\infty} \sum_{a=1}^{\infty} A_{p}^{(m)} A_{a}^{(m)}(n=p+q) .
$$

As a trial calculation, we have computed the first four eigenfunctions for the case

\begin{tabular}{|c|c|c|c|c|}
\hline$m$ & 1 & 3 & 5 & 7 \\
\hline$\lambda_{m}$ & -2.82776 & -32.1472 & -93.4775 & -186.781 \\
\hline$Q_{m}$ & +1.8489 & +0.4860 & +0.2407 & +0.1352 \\
\hline$p$ & $A_{p}^{(1)}$ & $A_{p}^{(3)}$ & $A_{p}^{(5)}$ & $A_{p}^{(7)}$ \\
\hline 1 & +1.00000 & +0.18615 & +.10564 & +.08356 \\
\hline 3 & -.02118 & +1.00000 & +.42332 & +.30054 \\
\hline 5 & -.00114 & -.15617 & +1.00000 & +.66385 \\
\hline 7 & -.00020 & -.00429 & -.35303 & +1.00000 \\
\hline 9 & -.00006 & -.00171 & +.01157 & -.62145 \\
\hline 11 & -.00002 & -.00062 & -.00327 & +.06758 \\
\hline 13 & -.00001 & -.00027 & -.00132 & -.00736 \\
\hline 15 & & -.00013 & -.00042 & -.00197 \\
\hline 17 & & -.00007 & -.00035 & -.00109 \\
\hline 19 & & -.00004 & -.00020 & -.00062 \\
\hline 21 & & -.00002 & -.00007 & -.00038 \\
\hline 23 & & -.00001 & -.00004 & -.00024 \\
\hline 25 & & & -.00003 & -.00016 \\
\hline 27 & & & -.00002 & -.00010 \\
\hline 29 & & & -.00002 & -.00007 \\
\hline 31 & & & & -.00005 \\
\hline 33 & & & & -.00004 \\
\hline 35 & & & & -.00003 \\
\hline
\end{tabular}
treated by Prins, Mulder and Schenk [1] and the results are shown in Table 1. In this

TABLE 1

case the term in $\alpha^{2}$ is neglected, but it has been verified that, in such cases in which this term must be included, the calculations are more rapid and less $A_{n}^{(m)}$ are required for each eigenfunction. In these cases, however, the dissipation term $\Phi(\xi, \eta)$ should be included in (1); the only effect of this is to introduce a known function of $n$ on the right hand sides of (7). It should also be pointed out that, in the computational process, it was not found necessary to use the Rayleigh principle in estimating the eigenvalues, although this would undoubtedly give more accurate estimates at any stage. 
Generalisation of the method. The method may readily be extended to the more general case of (1) where $u(\eta)$ may be any function satisfying Dirichlet's conditions. After appropriate transformation we should arrive at a modified (3a) with $\left(16 / \pi^{3}\right)$ $\left(y-y^{2} / \pi\right)$ replaced by some function $g(y)$ which must now be expanded as a Fourier cosine series in $(0, \pi)$.

$$
g(y)=\frac{1}{2} b_{0}+\sum_{a=1}^{\infty} b_{a} \cos q y \quad(q=1,2,3, \cdots) .
$$

The simultaneous differential equations can be formulated in terms of the coefficients, $b_{q}$ and the substitution (8) leads to the infinite determinant (11) where now

$$
a_{i j}=a_{i i}=\frac{1}{2}\left(b_{i+i}-b_{|i-i|}\right), \quad a_{i i}=f(\lambda, i)
$$

with

$$
f(\lambda, i) \equiv \alpha^{2} \lambda^{2}-\frac{1}{2}\left(b_{0}-b_{2 i}\right)-i^{2} .
$$

The infinite determinant $\Delta(\lambda)$ may be made convergent in the manner previously adopted [see footnote to Eq. (11)] provided that the sum of the off-diagonal terms $a_{i i}$ is absolutely convergent. If this is not the case, in fact provided only $\left|a_{i j}\right|$ is less than a positive constant, we may put $A_{n}=B_{n} / n^{2}$ in (8). and form the determinant for non-vanishing $B_{n}$, which can be made to converge as previously.

It is probable that the determination of the eigenvalues and the solution of the simultaneous equations may be performed in the manner described in the particular example, but the rapidity of convergence of the iterative procedures will of course depend upon the rate of decrease of the coefficients $b_{a}$ with increasing $q$. In any problem, approximations to the eigenvalues $\lambda_{m}$ may be determined independently from the $A_{n}^{(m)}$ by expanding $\Delta(\lambda)$ about the top left hand corner and taking into account sufficient columns and rows. As some check on the numerical work, this has been done in the specific example, taking into account four columns and rows. These yielded approximations to the first four $\lambda_{m}$ given by $\lambda_{1}=-2.82776, \lambda_{3}=-32.1482, \lambda_{5}=-93.4968$ and $\lambda_{7}=-227.164$. We do not consider other forms of the boundary conditions (3b) in this paper, except to point out that no significant change is involved by replacing $\vartheta(0, y)=1$ by $\vartheta(0, y)=$ a specified function of $y$. For example in the problem of flow between parallel plates, if the plates have respective temperatur $: s \pm \theta_{0}$ to the left of the origin, then we have $\vartheta(0, y)=$ $1-(2 y / \pi)$, and $\vartheta(x, y)$ may be represented by (4) with $n=2,4,6 \cdots$. It should also be pointed out that mathematically the method is basically equivalent to separation of variables and conversion of the resulting Sturm-Liouville equation to one of Hill's type [4]. The mode of solution is not the same, however, and no restrictions, save those stated, need be placed on $g(y)$.

\section{REFERENCES}

1. J. A. Prins, J. Mulder and J. Schenk, Appl. Sci. Research A, 2 (1951)

2. H. S. Carslaw and J. C. Jaeger, Conduction of heat in solids, Oxford, 1947

3. E. T. Whittaker and G. N. Watson, Modern analysis, Cambridge, 1927, p. 36

4. N. W. McLachlan, Theory and application of Mathieu functions, Oxford, 1947, p. 127 